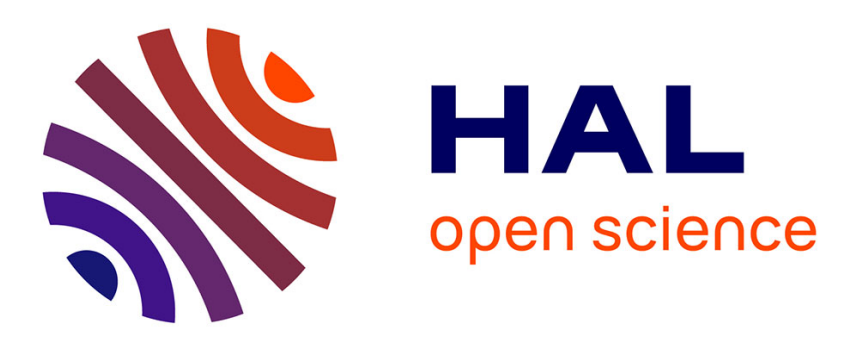

\title{
Francoise Salvadori et Laurent-Henri Vignaud Antivax. La résistance aux vaccins du XVIIIe siècle à nos jours Paris, Vendémiaire, 2019, 360 p.
}

\author{
Zoë Dubus
}

\section{- To cite this version:}

Zoë Dubus. Francoise Salvadori et Laurent-Henri Vignaud Antivax. La résistance aux vaccins du XVIIIe siècle à nos jours Paris, Vendémiaire, 2019, 360 p.. Annales. Histoire, Sciences sociales, 2020, pp.363-365. 10.1017/ahss.2020.148 . hal-03120749

HAL Id: hal-03120749

https://hal.science/hal-03120749

Submitted on 12 Apr 2021

HAL is a multi-disciplinary open access archive for the deposit and dissemination of scientific research documents, whether they are published or not. The documents may come from teaching and research institutions in France or abroad, or from public or private research centers.
L'archive ouverte pluridisciplinaire HAL, est destinée au dépôt et à la diffusion de documents scientifiques de niveau recherche, publiés ou non, émanant des établissements d'enseignement et de recherche français ou étrangers, des laboratoires publics ou privés. 
Dubus, Zoë. « Comptes rendus. Francoise Salvadori et Laurent-Henri Vignaud. Antivax. La résistance aux vaccins du XVIIle siècle à nos jours. Paris, Vendémiaire, 2019, 360 p. ", Annales. Histoire, Sciences Sociales, vol. 75e année, no. 2, 2020, pp. 363365.

Françoise Salvadori, docteure en virologie-immunologie, et LaurentHenri Vignaud, spécialiste d'histoire des sciences, mettent en lumière dans cet ouvrage la diversité des facteurs amenant des individus à refuser les vaccins, voire à se regrouper pour lutter contre cette pratique. L'essai peut être découpé en deux grandes parties: l'une historique, qui va au début du xviii ${ }^{e}$ siècle jusqu'aux années 1960 ; l'autre plus sociologique, qui tente d'analyser la résistance actuelle à la vaccination. L'espace géographique étudié se concentre sur l'Occident, bien que des allusions soient formulées sur le contexte colonial ou sur la médicalisation de pays en développement.

La technique de l'inoculation puis de la vaccination est une pratique médicale relativement récente au regard de l'histoire de la médecine occidentale. Elle apparaît en Angleterre au début du $\mathrm{xviii}{ }^{\mathrm{e}}$ siècle importée par Lady Montagu, une aristocrate anglaise qui l'a découverte à l'occasion d'un voyage à Constantinople. Dès son introduction, cette méthode fait l'objet d'une vive opposition tant de la part de certains membres du corps médical que d'une partie de la population. Les vaccins reposent en effet sur une logique scientifique différente de celle qui a prévalu durant des siècles et leur efficacité est alors difficilement démontrable (ils sont inoculés à des sujets sains en présupposant que ceuxci pourraient contracter une maladie). Susceptibles, en outre, de causer d'importants effets secondaires, voire de transmettre d'autres maladies, ils sont appréhendés avec méfiance.

Les pratiques médicales de la majorité de la population se caractérisent bien plus, à l'époque, par le recours à l'automédication et à des formes de médecines non officielles, mais administrées par des acteurs faisant partie de la communauté, qu'à des soins prodigués par un professionnel de santé, dont l'efficacité thérapeutique n'est pas, de fait, supérieure et dont le prix se révèle 
souvent prohibitif. La véritable lutte anti-vaccins s'organise à partir du $\mathrm{xix}^{\mathrm{e}}$ siècle, alors que certains États promulguent des lois destinées à contraindre les individus (les militaires puis les écoliers) à la vaccination. Ces ligues bénéficient de la mondialisation et des progrès de la presse pour diffuser largement leurs théories, inventant par là même des techniques de propagande comme l'envoi systématique de brochures aux jeunes parents. Elles se sont ensuite transformées et adaptées, jusqu'à nos jours, aux différents contextes médicaux, sociaux et techniques.

La mise en valeur de la riche iconographie sur laquelle les courants anti-vaccins se sont, tout au long de la période, largement appuyés pour défendre leur position aurait pu permettre d'alléger quelque peu le texte, qui s'avère parfois assez aride. L'utilisation des images ayant été un point fort de la lutte anti-vaccins, on peut s'étonner de constater que le cœur même de la rhétorique propre au thème de l'étude n'est pas analysé. Dans la même optique, on regrettera qu'une place si peu importante, pour ne pas dire inexistante, ait été laissée à la parole des acteurs et actrices des époques étudiées. Des citations plus nombreuses et un recours aux archives et aux témoignages auraient ainsi été bienvenus.

Cette réflexion nous amène à la question du traitement réservé à ces «antivax». Les auteurs laissent à plusieurs reprises percer un certain mépris, qu'il est regrettable de trouver dans un travail universitaire. Dans cet exemple, pris parmi d'autres, alors qu'ils abordent la question des discours prônant des «morales alimentaires » pour se prémunir des maladies sans avoir besoin de vaccins, les auteurs lancent: «On mange des graines comme les oiseaux pour réaffirmer notre proximité avec une nature idéalisée et rédemptrice » ( $\mathrm{p}$. 209). Si l'objectivité absolue n'est ni atteignable ni peut-être désirable, on attendrait toutefois une plus grande distance vis-à-vis des sujets d'étude.

Ce livre analyse cependant de manière fouillée toutes les thématiques de cette résistance, aussi qualifiée par l'Organisation mondiale de la santé d' « hésitation » vaccinale Elle connaît des fluctuations selon de nombreux facteurs: les périodes d'essor de la médecine sont favorables aux vaccins, qui sont dès lors partie intégrante d'un arsenal thérapeutique perçu comme performant; au contraire, lorsque la distance entre la population et le monde médical se creuse, les incompréhensions et la méfiance s'accentuent. Quand une médecine trop verticale tente de contraindre des individus mal informés sans prendre en considération leurs problématiques, la pratique vaccinale est rejetée. C'est le cas avec la mise en quarantaine au $\mathrm{xix}^{\mathrm{e}}$ siècle, qui empêche les gens de travailler et pose de graves problèmes économiques. Il en va de même dans les pays colonisés, où les campagnes 
vaccinales sont imposées sans tenir compte ni des pratiques médicales déjà à l'œuvre, ni des réalités de la vie quotidienne, ni de la culture propre à chaque territoire. Ces pratiques sont alors perçues comme violentes, autoritaires et donnent lieu à des rumeurs selon lesquelles les Occidentaux chercheraient, par exemple, à stériliser les populations locales.

La question de la vaccination obligatoire au nom de la santé publique, une notion qui se forge au cours du $x x^{\mathrm{e}}$ siècle, amène par ailleurs à de nombreuses évolutions législatives visant à protéger les droits des patients. La contrainte de la vaccination remet en effet en cause le droit de l'individu à être maître de son corps et à décider des traitements qu'il souhaite recevoir. Selon les législations, certaines résistances aux vaccins s'exercent à l'échelle nationale; la plus brûlante actualité nous permet d'en constater les continuités. Ainsi, l'Angleterre, paradoxalement à l'origine de la diffusion de la technique de la variolisation en Europe, connaît les mouvements anti-vaccins parmi les plus organisés et dont l'influence se poursuit jusqu'à nos jours. Dans l'épidémie actuelle de Covid-19, le gouvernement britannique a d'abord annoncé faire le choix de l' " immunité collective» avant de se raviser quelques jours plus tard. Cette notion d'immunité collective participe plus généralement d'un argument qui demeure aujourd'hui très puissant et qui ne cesse de se renforcer : celui de l'écologie, apparu à la fin du $\mathrm{xix}^{\mathrm{e}}$ siècle. Dès cette époque, des théories s'élaborent, en lien avec l'eugénisme alors en vogue, selon lesquelles l'organisme serait parfaitement capable de lutter contre les maladies à condition de recevoir une alimentation et une hygiène appropriées.

On est particulièrement frappé, à la lecture de ce livre, par l'opacité qui entoure les risques liés aux vaccins et par la volonté systématique des acteurs de les minimiser, voire de les dissimuler. Ce phénomène, qui peut être le fait du corps médical comme des gouvernements, s'applique à toutes les époques étudiées ; il va de la contagion par la syphilis à l'augmentation des cas d'autisme, plus récemment. Les dommages causés par cette opacité et par la difficile médiatisation des études cliniques dans la population peuvent être illustrés par l'exemple d'Andrew Wakefield. En 1998, ce médecin anglais affirme avoir découvert un lien entre la vaccination ROR (rougeoleoreillon-rubéole) et l'autisme. Bien qu'aucune équipe de recherche n'ait pu reproduire ses résultats, cette affaire, largement diffusée dans la presse, a eu un effet important sur la vaccination des enfants, notamment en Angleterre, pays dans lequel on estime avoir perdu une dizaine d'années de couverture vaccinale sur la rougeole. Or, il se trouve que Wakefield avait 
non seulement été engagé à hauteur de 61000 euros afin de produire ces résultats, mais qu'il avait, de surcroît, déposé un brevet quelques mois plus tôt pour un autre vaccin contre la rougeole.

D'autres affaires similaires, également très médiatisées, ont largement apporté de l'eau au moulin des opposants affirmés de la vaccination. En instillant, de manière récurrente, l'idée d'un lien entre maladies graves et vaccins, elles sont déterminantes pour expliquer les réticences d'une partie de la population. Pour la période contemporaine, cette hostilité doit se comprendre dans un contexte plus général de méfiance à l'encontre de la médecine: chaque génération ou presque, depuis plusieurs décennies, a connu son scandale sanitaire (de la Thalidomide, dans les années 1960, au Médiator, en passant par l'affaire du sang contaminé, pour ne citer qu'eux). L'argument économique sert aussi de repoussoir depuis la diffusion à grande échelle de la technique. Jusque dans les années 1960, les laboratoires nationaux fabriquaient encore de façon assez artisanale les vaccins. Dans l'impossibilité de faire face aux procès qui leur sont intentés et devant la complexité, toujours accrue, des divers contrôles auxquels sont soumis les médicaments, ces compagnies cessent progressivement la production vaccinale et laissent la place à quelques grands groupes pharmaceutiques, qui se partagent dès lors le marché. Ce contexte réactive l'ancienne accusation de la vénalité des médecins vaccinateurs, qui existait aux xviii ${ }^{\mathrm{e}}$ et $\mathrm{xix}^{\mathrm{e}}$ siècles pour l'adapter à une nouvelle figure repoussoir : les lobbys pharmaceutiques, ou «Big Pharma ». Enfin, les mouvements antivaccins ont récemment trouvé un nouveau souffle grâce à Internet et, en particulier, aux réseaux sociaux. Les protagonistes de ces courants y diffusent à grande échelle des contenus visant un public à la fois de plus en plus concerné par les enjeux de santé et décidé à exercer un contrôle croissant, qui passe notamment par une critique de la médecine contemporaine perçue comme trop paternaliste, déshumanisante et autoritaire.

Cet ouvrage met en lumière la diversité et l'extrême complexité des résistances-hésitations face aux vaccins, qu'il est impossible de réduire à une cause telle que la croyance religieuse, une opinion que les auteurs déconstruisent en soulignant les discours favorables des grandes religions envers la vaccination, conçue comme un acte de responsabilité collective. En revanche, de petits groupes religieux ou alternatifs, en marge de la société, opposés à la vaccination, comme les membres des écoles Steiner, peuvent constituer des foyers de diffusion des maladies. Plus que l'appartenance religieuse, c'est ici une corrélation de causes (rejoignant en partie celles de l'écologie) qui amènent ces types de population à la non-vaccination. D'une grande densité d'informations, ce travail représente 
une somme de connaissances sur un phénomène complexe et pointe, sur près de trois siècles, la constance des arguments tout comme leur évolution. Les lecteurs y trouveront des pistes de réflexions à poursuivre, notamment sur les questions de genre ou d'histoire coloniale. 